\title{
Gert-Joachim Glaeßner
}

\section{»Totalitarismus « - Reflexionen zu einer wissen- schaftlichen und politischen Debatte}

1989 schien das Ende des »Zeitalters der Extreme« (Hobsbawm 1994) zu markieren, und viele Beobachter sahen in der »dritten Welle der Demokratisierung « (Huntington 1991) Anzeichen für einen endgültigen Sieg der Demokratie, wenn nicht gar ein »Ende der Geschichte«. Verwundert rieben sich viele Zeitgenossen die Augen und fragten sich angesichts des kläglichen Endes des Kommunismus, warum man sich so sehr vor ihm gefürchtet oder warum viele ihn so sehr bewundert und unterstützt hatten.

Nach dem gewaltsamen Ende des Nationalsozialismus und Faschismus 1945 erschien die Bipolarität der Welt ein unabwendbares und nicht veränderbares Schicksal der modernen Staatenwelt mit tiefgreifenden Konsequenzen für das innere Gefüge und das Selbstverständnis der Staaten und politischen Systeme. Alternativen, die sich als »Dritte Wege« zwischen Kapitalismus und Sowjetsozialismus, liberaler Demokratie und Diktatur des Proletariats zu behaupten versuchten, hatten kaum eine Chance. Die liberalen Demokratien, die erst nach dem Ersten Weltkrieg mit Einführung des allgemeinen Wahlrechts, der Parteiendemokratie und einer sich entwickelnden politischen Öffentlichkeit die von ihnen postulierten normativen Standards auch faktisch erreichten, sahen sich nicht nur durch die Kräfte des ancien regime, sondern auch durch zwei historisch neue Massenbewegungen in die Zange genommen: Den Kommunismus der Bolschewiki mit seiner Strategie der Weltrevolution, gesteuert von der Komintern, und den faschistischen Bewegungen. Beiden »politischen Mythologien « (Furet 1998: 13) wohnte eine heute kaum noch verstehbare und nachvollziehbare Kraft inne. François Furet hat von einer »magischen Aura « gesprochen, die beide Bewegungen umgeben und die revolutionären Leidenschaften der Massen geschürt habe.

Eine praktisch geltend gemachte, teleologisch ausgerichtete Ideologie und Terror gegenüber denen, die sich ihren Glaubenssätzen nicht anschlossen oder sie offen bekämpften, konstituierten, wie Hannah Arendt im letzten Kapitel ihrer Studie über die Elemente und Ursprünge totaler Herrschaft 
schrieb, eine neue Herrschaftsform, die mit den Kategorien der klassischen, auf Aristoteles zurückgehenden Typologien politischer Herrschaft nicht mehr hinreichend zu analysieren sei. Diese neuartigen politischen Bewegungen und die von ihnen gegründeten Herrschaftsordnungen hatten mit einer wesentlich größeren Radikalität als frühere autoritäre Regime die Verbindungen zur liberalen Tradition politischen Denkens zu zerschlagen versucht. Gemeinsam war ihnen eine auf der Nutzung der modernen technischen Mittel und der Massenmobilisierung basierende Herrschaftspraxis, die dann auch in der ersten Phase der theoretischen Auseinandersetzung mit dem Kommunismus und dem Faschismus bzw. Nationalsozialismus im Vordergrund stand. Dies unterschied die neuen Diktaturen des 20. Jahrhunderts von früheren Vorläufern und seien sie, wie z. B. die »Schreckensherrschaft « der Jakobiner, auch noch so radikal gewesen. Die Wissenschaft hatte es also seit dem Aufkommen der großen Massenbewegungen unseres Jahrhunderts mit einem historisch neuartigen Phänomen zu tun: mit dem $\gg$ Totalitarismus «.

Im Rückblick auf die Geschichte des 20. Jahrhunderts erscheinen der Bolschewismus Stalinscher Prägung und der Nationalsozialismus als die beiden fundamentalen Herausforderungen für eine auf der Idee der unveräuBerlichen Menschenrechte gründende freiheitliche Gesellschaft. Als unversöhnliche Gegner der Demokratie waren die faschistischen und bolschewistischen Bewegungen und Regime vergleichbar, als Machtordnungen hatten sie viele Ähnlichkeiten. Selbst ihre Ideologien hatten, bei allen fundamentalen Unterschieden und Traditionsbezügen, ihren Absolutheitsanspruch gemein. Der Kommunismus jedoch zielte auf eine völlige Umwälzung der alten politischen und sozialen Ordnung, während der Faschismus und der Nationalsozialismus auf die totale Zerstörung der politischen Ordnung aus waren. Die Singularität des in dieser Hinsicht unvergleichbaren Nationalsozialismus besteht in der ideologisch begründeten Absicht und der unter Nutzung moderner Organisation und Technik realisierten physischen Vernichtung der »jüdischen Rasse « und anderer als »minderwertig « angesehener Bevölkerungsgruppen.

Viele Diskussionen in den letzten Jahren haben deutlich werden lassen, daß der Begriff »Totalitarismus « zu einem Catchword geworden ist, das für ein bestimmtes allgemeines und diffuses Bild, nicht aber für eine theoretisch und politisch anspruchsvolle Reflektion dieses Jahrhundertphänomens steht. Daher möchte ich im folgenden auf die ideengeschichtlichen und politisch-theoretischen Grundlagen der Totalitarismustheorie eingehen, ihre Leistungen und Grenzen reflektieren und schließlich in aller Kürze ihre Brauchbarkeit für eine empirische Analyse kommunistischer Systeme wie dem der DDR diskutieren. 


\section{Entwicklungslinien des Totalitarismusbegriffs}

Mit dem Ende des Kommunismus haben die Schriften der Sozialphilosophin Hannah Arendt nach Jahrzehnten der Vergessenheit neue Aufmerksamkeit gefunden. Auch wenn man sich des Eindrucks nicht erwehren kann, daß viele, die sich auf sie berufen, ihr Hauptwerk über die Elemente und Ursprünge totaler Herrschaft allenfalls oberflächlich zur Kenntnis genommen haben, wird sie immer wieder als »Kronzeugin « für die totalitäre Natur des Sozialismus sowjetischen Typs herangezogen. Festzuhalten ist, daß sich die geschichtsphilosophisch begründete Erklärung der eschatologischen Massenbewegungen des 20. Jahrhunderts bei Arendt nicht ohne weiteres für eine empirische Analyse der Systeme sowjetischen Typs anwenden ließ. Dies war auch der Grund dafür, daß in der Verwendung totalitarismustheoretischer Ansätze meist auf normativ und institutionentheoretisch argumentierende Typologien zurückgegriffen wurde, wie sie von Carl Joachim Friedrich, Raymond Aron und anderen vorgelegt worden sind (Aron 1970; Friedrich 1957; zur Entwicklung der Totalitarismustheorie vgl. Glaeßner 1982 u. 1995).

Totalitarismus war für Hannah Arendt eine Herrschaftsform, die mittels permanenten Terrors in Bewegung gehalten wird und die auf den Terror angewiesen ist. Der Terror stelle nicht, wie in früheren Tyranneien, Mittel zu einem Zweck dar, sondern finde in sich selbst Genüge und entziehe sich daher allen rationalisierbaren sozialen und ökonomischen Kriterien.

»Das Wesen totalitärer Herrschaft ... ist der Terror, der aber nicht willkürlich und nicht nach den Regeln des Machthungers eines einzelnen (wie in der Tyrannis), sondern in Übereinstimmung mit außermenschlichen Prozessen und ihren natürlichen oder geschichtlichen Gesetzen vollzogen wird. Als solcher ersetzt er den Zaun des Gesetzes, in dessen Umhegung Menschen in Freiheit sich bewegen können, durch ein eisernes Band, das die Menschen so stabilisiert, daß jede freie, unvorhersehbare Handlung ausgeschlossen wird. Terror in diesem Sinne ist gleichsam das 'Gesetz', das nicht mehr übertreten werden kann. ... Der Terror ist nicht ein Mittel zu einem Zweck, sondern die ständig benötigte Exekution der Gesetze natürlicher oder geschichtlicher Prozesse.« (Arendt 1986: 711)

Die gemeinsame erbitterte Gegnerschaft gegenüber den Ideen der individuellen Menschen- und Bürgerrechte und der modernen Demokratie, die Mittel des Kampfes um die Macht und der nach der Machtübernahme ins Werk gesetzte Massenterror drängten schon früh die Frage nach Gemeinsamkeiten von Nazismus und Kommunismus auf. Waren sie vorübergehend oder dauerhaft? War der eine Totalitarismus die Reaktion auf den anderen, wie nicht nur Ernst Nolte gemutmaßt hat? Vor allem aber galt es eine Antwort auf die Frage zu finden, ob es einen wertneutralen Zugang zum Verständnis des Totalitarismus überhaupt geben könne. Muß nicht, so fragt Pierre Hassner, ein Nicht-Ideologe zu einem Ideologen werden, um das andere, fremde System überhaupt begreifen zu können? Besteht möglicherweise der einzige Weg, den Totalitarismus zu begreifen darin, anti-totalitär zu 
sein, und kann man dies sein, ohne eine »anti-ideologische Ideologie« $\mathrm{zu}$ kreieren, gleichsam einen »anti-totalitären Totalitarismus« (Hassner 1985: 17)?

Dies waren die Fragen, die die »Renegaten « des Kommunismus wie Franz Borkenau, Arthur Koestler und George Orwell, die Intellektuelle und Schriftsteller wie Albert Camus oder Ignazio Silone und Sozialwissenschaftler wie Raymond Aron und Richard Löwenthal schon in den Jahren nach den Moskauer Prozessen der 30er Jahre bewegt haben. Ihre Antworten fielen höchst unterschiedlich aus. Dies sind auch die Fragen, die nach dem Ende des Kommunismus erneut so kontrovers diskutiert werden, als ginge es immer noch darum, die säkulare Herausforderung der Demokratie hier und jetzt geistig zu bekämpfen.

Totalitarismustheoretische Konzepte waren das wissenschaftliche Pendant einer politischen Auseinandersetzung, die bis in die Zeit des I. Weltkrieges zurück reicht, die die Zwischenkriegszeit, die Zeit des II. Weltkrieges, die Nachkriegszeit und die Jahre bis 1989, das heißt die längste Zeit unseres Jahrhunderts bestimmt hat. Der Totalitarismus erfüllte für die durch die Herausforderungen der faschistischen, des nationalsozialistischen und des stalinistischen Systems um ihr Selbstverständnis und die angemessene »Gegenideologie « ringenden westlichen Demokratien in den 30er, 40er und besonders in den 50er Jahren eine willkommene Integrationsfunktion. Der Begriff des Totalitarismus lieferte den an Systematisierung und begrifflicher Abstraktion interessierten Vertretern der Sozialwissenschaften eine prima vista bündige Erklärung für ein Phänomen, das 1938 Paul Valéry zu dem zynisch-resignierenden Ausspruch veranlaßt hatte, es sei bemerkenswert, »daß die Diktatur jetzt so ansteckend sei wie einst die Freiheit« (Hildebrandt 1968: 397)

Es hat allerdings nie die eine Totalitarismustheorie gegeben. Sowohl die wissenschaftstheoretischen als auch die politisch-weltanschaulichen Positionen der »Totalitarismustheoretiker « waren höchst verschieden, oft konträr. In einem wesentlichen Punkt aber gab es Übereinstimmung: Der Totalitarismus wurde als eine »moderne « Variante der Diktatur begriffen, die durch politische Radikalität, umfassenden Regelungsanspruch, ideologischen Wahrheitsanspruch und Unversöhnlichkeit sowie durch die technische Verfeinerung des Herrschaftsinstrumentariums von traditionellen Autokratien zu unterscheiden sei und eine neue Variante der Typologie der Herrschaftsformen darstelle. Diesen Aspekt haben vor allem Carl Joachim Friedrich und Zbigniew Brzezinski, später in ähnlicher Weise Raymond Aron und Peter Graf Kielmansegg unterstrichen. Diese Modelle hoben bestimmte Strukturmerkmale wie ein Einparteiensystem, ein Informationsmonopol oder die Rolle der Geheimpolizei hervor, die in ihrer Kombination typischerweise totalitäre von autoritären Systemen unterschieden. Da 
die genannten Elemente aus der Analyse des Nationalsozialismus und des Stalinismus gewonnen waren und wesentlich auf die Statik dieser Herrschaftsform abhoben, erschienen sie der Mehrzahl der Kommunismusexperten nicht mehr zur Analyse post-stalinistischer Systeme geeignet, die sich in vielerlei Hinsicht veränderten und bestimmte als konstitutiv angenommene Elemente des Totalitarismus, wie den Massenterror, abstreiften.

Ähnliche Vorbehalte mußten gegen Modelle geltend gemacht werden, die eher die dynamischen Aspekte des Totalitarismus in den Mittelpunkt stellten. Auch sie hatten die totalitären Diktaturen der 30er und 40er Jahre im Blick. Bei Sigmund Neumann waren es der Allmachtsanspruch und die Maßlosigkeit in bezug auf die Zeit und den Wirkungsgrad der Herrschaft (»Tausendjähriges Reich« und kommunistische Zukunftsgesellschaft), die den Totalitarismus nicht nur zu einer permanenten Herausforderung für die Demokratie, sondern auch zu einer Spielart der Diktatur machten, die darauf angelegt sei, ihre Schöpfer zu überleben. Anders als die tradierten persönlichen Autokratien sei der Totalitarismus auf Dauer gestellt und müsse daher den Anspruch erheben, alle Bereiche des menschlichen Lebens seiner Herrschaft und Kontrolle zu unterwerfen (Neumann 1965: 3). Die modernen Diktaturen seien darauf angewiesen, die Gesellschaft als im Kriegszustand befindlich zu verstehen, da nur so ihr »Kampfgeist « aufrechterhalten werden könne. Totalitäre Herrschaft sei nur als »permanente Revolution «, als permanenter Ausnahmezustand vorstellbar, sie durchdringe als totale Revolution alle Bereiche und Handlungen des politischen Organismus (Neumann 1965: XV).

Den von Neumann konstatierten Allmachtsanspruch führte Hannah Arendt auf ideologische Grundannahmen dieser Bewegungen zurück, die Vorstellung nämlich, sich im Einklang mit den Gesetzen der Natur (Rassenlehre) oder der Geschichte (Marxismus-Leninismus) zu befinden. Die Verachtung der totalitären Machthaber speise sich aus dem Anspruch, einzig berufene Interpreten dieser Gesetze zu sein. Der explizit formulierte Alleinvertretungsanspruch einer politischen Führungsgruppe, an Stelle der Bürger zu bestimmen, was das allgemeine Wohl sei, weil man das ideologische Instrumentarium zu besitzen meint, um diese Definition des Gemeinwohls verbindlich vornehmen zu können, bedeutet die dauerhafte, nicht nur temporäre Ausschaltung der Bürger aus dem politischen Prozeß. Aus diesem ideologischen Anspruch, den Hannah Arendt als vermeintliches Wissen um die Gesetze der Natur oder der Geschichte beschrieben hat, erwächst der Wille, nicht nur die politische, sondern auch die soziale Sphäre umfassend zu regulieren und zu kontrollieren, d. h. gleichzuschalten.

Totalitarismus ist eine Erscheinung des modernen Massenzeitalters, die aus der Entfremdung der Menschen von ihren natürlichen und sozialen Lebensbedingungen erwächst. Die totalitäre Versuchung besteht im Verspre- 
chen, diese Entfremdung zu überwinden, sei es in der Volksgemeinschaft, dem »estato corporativo « oder der kommunistischen Gesellschaft. Gemeinsam waren dem Totalitarismus der 30er und 40er Jahre historisch neue Herrschaftsformen und -techniken, die das bis dahin gekannte Maß an Brutalität und herrschaftstechnischer Perfektion, aber auch die ZweckMittel-Relationen »klassischer « Diktaturen sprengten. Das historisch Neuartige an diesen Herrschaftssystemen führte Carl Joachim Friedrich zu der Auffassung, »daß die totalitäre Diktatur historisch einzigartig und sui generis ist und daß aufgrund der uns jetzt vorliegenden Tatbestände behauptet werden kann, daß die faschistischen und kommunistischen totalitären Diktaturen in ihren wesentlichen Zügen gleich sind, d. h., daß sie sich untereinander mehr ähneln als anderen Systemen staatlicher Ordnung, einschließlich älterer Formen der Autokratie« (Friedrich 1957: 15).

Die These, daß der Totalitarismus eine politische Ordnung sui generis sei und daß totalitäre Systeme sich wesentlich gleich sind, hatte Friedrich bereits auf der Totalitarismuskonferenz von 1953 vorgetragen. Dort argumentierte er eher »statisch « und verquickte zudem die Analyse bestehender »bolschewistischer « mit der bereits untergegangener faschistischer Systeme, eine Vorgehensweise, die es erschwerte, Entwicklungen der noch bestehenden »totalitären « Ordnungen zu bewerten. Später hat Friedrich die These, daß faschistische und kommunistische totalitäre Diktaturen in wesentlichen Zügen gleich seien, mehrfach modifiziert und abgewandelt. In der zweiten revidierten Auflage von Totalitarian Dictatorship and Autocracy heißt es nur noch, daß sie sich im Grunde ähnlich seien, an anderer Stelle ist davon die Rede, daß sie »im Grunde gleich «, aber nicht »völlig gleich« seien (Friedrich 1966: 46).

Andererseits wies Friedrich auf signifikante Unterschiede zwischen faschistischen und kommunistischen Diktaturen hin. In Politik als Proze $\beta$ der Gemeinschaftsbildung (englisch: Man and His Government) von 1963 konstatierte er einen »scharfen Gegensatz zum faschistischen Totalitarismus «, als er dem kommunistischen Totalitarismus konzedierte, daß er »gegenüber kulturellen Verschiedenheiten « eine »erhebliche Toleranz gezeigt « habe. Dies bedeutete allerdings keine Annäherung an demokratische Vorstellungen von politischem Pluralismus, sondern sei »die Konsequenz aus der Abwertung kultureller Werte. Die herrschende materialistische Ideologie ist der Grund für die vergleichsweise erhebliche Indifferenz gegenüber allen kulturellen Traditionen « (Friedrich 1970).

Normativer Ausgangspunkt der Totalitarismusanalyse war der Widerstand gegen den Faschismus bzw. Nationalsozialismus und den Leninismus bzw. Stalinismus. Die Verbrechen des Nationalsozialismus und die Schreckensherrschaft des Stalinismus der 30er Jahre, die nach 1945 wieder aufzuleben schien und zudem nach Mittel-Osteuropa exportiert worden war, konnten 
nur als fundamentaler Angriff auf die grundlegenden Ideen der Menschenwürde und der persönlichen Freiheit begriffen werden. Die Gefährdung der Demokratie und der totale Zugriff auf das persönliche Leben der Menschen durch einen neuen Typus der Diktatur stellten die Herausforderung in der ersten Hälfte des 20. Jahrhunderts dar. Vom Bestreben geleitet, die Kontrolle über die Massen zu erlangen und zu erhalten, d. h. »mittels Zwangsorganisationen, die von einer monopolistischen Staatspartei manipuliert werden «, haben diese Diktaturen alle Lebensbereiche durchdrungen - insofern waren sie ein »totalitäres «, nämlich allumfassendes Gegenbild zu pluralistischen Gesellschaften (Fraenkel 1991: 297 ff.). ${ }^{1}$ Intentionalität, Technizität und ideologische Fundierung sind die wesentlichen Elemente, die totalitären Regimen als Gegenpole zum europäischen liberalen und demokratischen Denken zugeschrieben werden, wobei gelegentlich als viertes Element der Aspekt der Historizität ins Spiel kommt.

\section{Totalitarismus, Kommunismus und Terror}

Eine zentrale Rolle in allen Totalitarismustheorien aber spielt der Massenterror als unverzichtbares Element totalitärer Herrschaft. Während bei Friedrich zivilisationskritische Töne anklangen, die den Terror als eine Art Zwischenstadium zur Forcierung einer formierten Massengesellschaft totalitären Zuschnitts erscheinen lassen, argumentierte Hannah Arendt geschichtsphilosophisch. Terror als Wesensmerkmal totalitärer Herrschaft sei das Ergebnis eines Selbstverständnisses der Machthaber, die von sich behaupten, das »Gesetz der Geschichte« oder das »Recht durch Natur « zu exekutieren, und die deshalb glauben, auf das positive Recht, das ja »immer nur konkrete Ausgestaltung einer höheren Autorität zu sein behauptet«, verzichten zu können.

»Dem Glauben der Nazis an Rassegesetze lag die Darwinsche Vorstellung vom Menschen als einem eigentlich zufälligen Resultat einer Naturentwicklung zugrunde, die nicht notwendig

1 An diese Überlegungen knüpfte Karl Dietrich Bracher in den 80er Jahren an. »Wie immer verfassungs- und ideologietheoretisch der Begriff des Totalitarismus gefaßt werden mag, gemeinsam war den großen antiliberalen Ideologien das Streben nach Monokratie oder Oligarchie im Namen der Volksherrschaft und die Vernichtung der Opposition. Willkür und Gewalt werden, wo nicht überhaupt als unumgänglich im Geschichtsprozeß angesehen, mit dem höheren Recht der Revolution oder des neuen Gemeinschafts- und Herrschaftsprinzips sanktioniert. Dazu kommt der Anspruch, den unumgänglichen Gesellschaftswandel durch Organisierung, Mobilisierung, Erfassung der gesamten Bevölkerung planvoll zu gestalten und die modernen technischen Mittel, die den Diktaturen des 20. Jahrhunderts ein so viel wirksameres Instrumentarium der Kontrolle und des Zwangs verfügbar machten, voll einzusetzen: neben Bürokratie und Polizei die weitverzweigten Organisationen der Partei, aber auch der Justiz und des Bildungswesens. Wieweit dies gelingt und zur Realität wird, ist eine andere Frage. Aber intentionell und potentiell ermöglicht der technische Fortschritt solchen Diktaturen - und ihren Gesellschafts- und Herrschaftsideologien - über den politischen Bereich hinaus den Anspruch auf monokratische Erfassung der gesamten Gesellschaft.«(Hervorh. GJG; Bracher 1985: 164) 
mit dem Menschen an ihr Ende gekommen zu sein braucht. Dem Glauben der Bolschewisten an Geschichtsgesetze liegt Marx' Vorstellung von der menschlichen Gesellschaft als dem Resultat eines gigantischen Geschichtsprozesses zugrunde, der mit immer vergrößerter Geschwindigkeit seinem Ende entgegenrast und sich selbst als Geschichte aus der Welt schafft. ... Auffallend ist, daß - so verschieden diese beiden Ideologien voneinander sind, so großartig erfüllt mit den besten abendländischen Traditionen der dialektische Materialismus, so kläglich-vulgär, wiewohl auf einem echten Erfahrungselement basierend, der Rassismus - in beiden Konzeptionen das Bewegungsgesetz sich gleich äußert: Es läuft in jedem Falle auf ein Gesetz der Ausscheidung von 'Schädlichem' oder Überflüssigem zugunsten des reibungslosen Ablaufs einer Bewegung hinaus, aus der schließlich gleich dem Phönix aus der Asche eine Art Menschheit erstehen soll. Würde das Bewegungsgesetz in positives Recht übersetzt, so könnte sein Gebot nur heißen: Du sollst töten! Die Ideologien ziehen diese Schlußfolgerung nicht, weil sie noch damit rechnen, daß der Prozeß irgendwann einmal an sein Ende kommen wird, etwa wenn die klassenlose Gesellschaft auf der ganzen Erde verwirklicht oder die Herrenrasse über die ganze Welt zur Herrschaft gekommen ist.« (Arendt 1986: 707f)

Der Glaube an Gesetzmäßigkeiten führt nach Auffassung Arendts aber keineswegs zu einer abwartenden Haltung der Bewegungen. Sie warten nicht darauf, daß sich diese Gesetzmäßigkeiten »von selbst « durchsetzen, sie setzen vielmehr alles daran, diesen Prozeß durch das Instrument des Terrors zu beschleunigen:

»Das eiserne Band des Terrors konstituiert den totalitären politischen Körper und macht ihn zu einem unvergleichlichen Instrument, die Bewegung des Natur- oder Geschichtsprozesses zu beschleunigen. ... Die an sich notwendig ablaufenden Prozesse will der Terror auf eine Geschwindigkeit ... bringen, die sie ohne die Mithilfe der zu einem Menschen organisierten Menschheit nie erreichen könnten. Praktisch heißt dies, daß Terror die Todesurteile, welche die Natur angeblich über 'minderwertige Rassen' und 'lebensunfähige Individuen' oder die Geschichte über 'absterbende Klassen' und 'dekadente Völker' gesprochen hat, auf der Stelle vollstreckt, ohne den langsameren und unsicheren Vernichtungsprozeß von Natur oder Geschichte selbst abzuwarten.« (Arendt 1986: 714)

Diese Beschleunigung des Entwicklungsprozesses mit Hilfe des Terrors steht für Arendt in einem bemerkenswerten Widerspruch von Bewegung und Bewegungsziel, denn diese Form der Bewegung ist ihrer Theorie zufolge nicht zielgerichtet. Sie ist vielmehr Bewegung um der Bewegung willen. Sie ist notwendig, um zu verhindern, daß ein Ziel erreicht wird, welches die totalitäre Herrschaft selbst überflüssig machen würde. Bewegung erfordere das Handeln vieler. Nicht so im Totalitarismus. Er kenne kein eigentliches Prinzip des Handelns, da der Mensch nur Material der über ihn waltenden Prozesse, der Gesetze von Natur und Geschichte sei, die die totalitäre Bewegung zu verwirklichen vorgebe. Das Verlangen nach Handeln werde ersetzt durch ideologische Präparierung.

Die wesensmäßigen Gemeinsamkeiten von Nationalsozialismus, Faschismus und Kommunismus lagen für Hannah Arendt also tiefer. Terror statt Geltung des positiven Rechts ist für sie mehr als eine »Zweckmäßigkeit«, die unter gewandelten Bedingungen auch abgeschafft werden kann, er ist der notwendige Ausdruck einer Natur- oder Geschichtsphilosophie, die, wenn sie sich politisch formiert, zwangsläufig zu totalitären Formen $\mathrm{Zu}$ flucht nehmen muß. Solange totalitäre Bewegungen nicht an der Macht 
sind, seien sie nicht genötigt, den »Beweis« für die Richtigkeit ihrer als gesichert ausgegebenen Voraussagen anzutreten. Sie könnten und müßten sich damit begnügen, mit Hilfe der Propaganda jene »Emanzipation des Denkens von erfahrener und erfahrbarer Wirklichkeit « zustande zu bringen, die sie, einmal an der Macht, mit Hilfe des Terrors durchsetzen. Die geistige Manipulation der Menschen besorge die Ideologie, die ein geschlossenes System der Erklärung jener »objektiven« Gesetzmäßigkeiten sei, der Herrscher und Beherrschte, Vollstrecker und Opfer unterworfen sind. Obwohl nur diejenigen Ideologien als totalitär erscheinen, die - wie der »Rassismus « und »Kommunismus « - von totalitären Bewegungen voll entwickelt worden seien, meint Hannah Arendt, daß alle Ideologien »totalitäre Elemente« enthalten und daß dieses »eigentliche Wesen aller Ideologien «, das in ihrem Anspruch wurzele, Gesamtdeutungen von Natur- und Gesellschaftsprozessen vorzunehmen, in dem Augenblick sichtbar werde, wenn sie vom totalen Herrschaftsapparat genutzt werden (Arendt 1986: 740ff).

Auch bei dem neben Hannah Arendt wohl einflußreichsten Totalitarismustheoretiker Carl Joachim Friedrich ist der Terror konstitutives Element jeder totalitären Herrschaft. Allerdings wird seine zentrale Bedeutung anders, nämlich institutionentheoretisch begründet. In seinem Referat auf der Totalitarismus-Tagung 1953 hatte Friedrich von einem System terroristischer Polizeikontrolle gesprochen, es aber abgelehnt, die Existenz einer terroristischen Geheimpolizei, die der Armee den Rang abläuft, als konstitutives Merkmal jedes totalitären Systems zu kennzeichnen. Ob die Geheimpolizei überhaupt eine alles dominierende Stellung erringen werde, sei völlig offen. In der englischen zweiten Auflage des Buches 1965, das von Friedrich und Brzezinski gemeinsam verfaßt wurde, war ganz allgemein von einem »Terrorsystem auf physischer und psychischer Grundlage« die Rede. In der deutschen Fassung seines Buches hatte Friedrich 1957, trotz der sich bereits zu dieser Zeit deutlich abzeichnenden Abkehr von den terroristischen Methoden stalinistischer Herrschaftsausübung, das Anwachsen des Terrors als konstitutives Element totalitärer Machtausübung gekennzeichnet:

\footnotetext{
»Der Totalitäre ist überzeugt, daß die Massen auf seiner Seite sind oder es jedenfalls sein sollten. In jedem Fall müssen sie gegen den Feind verteidigt werden, der sich bemüht, die Indoktrination hintanzuhalten, der selbst auf den Sturz des totalitären Systems sinnt. Dieser Kampf gegen die Feinde des Volkes dauert ununterbrochen an und ist ... um so intensiver, je gefestigter und sicherer das totalitäre Regime dasteht, denn es kann sich nun größere Gewalttätigkeiten erlauben, und die anfängliche Geduld und Vorsicht weichen dem ungezügelten Terror.« (Friedrich 1957: 57)

Während Friedrich, ähnlich wie Arendt, ursprünglich von einer permanenten Verschärfung des Terrors ausging, sah er in späteren Veröffentlichungen nicht nur eine Relativierung des Terrors, sondern sogar Ansätze einer Konsensbildung in »totalitären« Gesellschaften.
} 
»Ein derartiger Konsensus schafft eine Basis, auf der andersartige Verfahren möglich werden, als sie gegenüber einer in breitem Ausmaß feindselig eingestellten Bevölkerung angewandt werden müssen. Solche Verfahren waren der Kern dessen, was man Chruschtschows Popularismus genannt hat, durch den er vor allem die niederen Kader und die breite Masse der Parteimitglieder aktiviert und die Beteiligung der Bevölkerung angesprochen hat.« (Friedrich 1966: 50f)

Es sei das Wesen totalitärer Herrschaft, so argumentierte Friedrich, ähnlich wie Hannah Arendt, daß sie es unternehme, »alle ihr unterworfenen Personen $\mathrm{zu}$ atomisieren, d. h. in Massenmenschen $\mathrm{zu}$ verwandeln.« Diese Art entpersönlichter Homunculus sei allerdings »auch sonst im Verlauf der Industrialisierung und Massenproduktion« eine verbreitete Erscheinung. Als solcher werde er zum »wichtigsten Vorkämpfer totalitärer Bewegungen« (Friedrich 1970: 480).

\section{Totalitarismus und Post-Stalinismus}

Systeme, die mit einem totalitären Anspruch angetreten sind, waren vielfältigen Veränderungstendenzen und -notwendigkeiten unterworfen, über deren Zielrichtung nur schwer zuverlässige Aussagen möglich waren. Geschlossene Modelle, die die Unveränderbarkeit totalitärer Systeme unterstellten, erwiesen sich nach dem Ende des Stalinismus für eine empirische Analyse sich offenkundig verändernder politischer und gesellschaftlicher Ordnungen als nur noch bedingt brauchbar. Offene Modelle, wie sie u.a. von Leonard Shapiro (1969) oder Juan Linz (1975) entwickelt worden sind, oder Versuche wie die von Peter-Christian Ludz (1970), genauer zwischen Autoritarismus und Totalitarismus zu unterscheiden und den Totalitarismusbegriff offener zu formulieren, boten hingegen die Chance, ihn aus seinem politischen Kontext und seiner Verankerung in der Lehre der Herrschaftsformen zu befreien.

Daß diese Chance nicht ergriffen wurde, hing m. E. mit zwei Faktoren zusammen. Zum einen war der Begriff theoriegeschichtlich »besetzt«. Sowohl die statischen Modellbildungen in der Tradition Friedrichs als auch die dynamischen Vorstellungen des Totalitarismus in der Nachfolge von Hannah Arendt waren zu sehr in der Tradition der Typologie der Herrschaftsformen verwurzelt, als daß sie für eine politisch-soziologische Analyse sich verändernder und wandelnder politischer und sozialer Systeme brauchbar gewesen wären. Zum anderen war der Begriff politisch aufgeladen - und ist es immer noch -, was dazu führte, daß seine Verwendung mehr auf die politische Signalwirkung als auf seine empirische Leistungsfähigkeit abzielte.

Diese Einschränkungen bedeuten jedoch keineswegs, daß auf einen Vergleich von Diktaturen aus politischen Gründen verzichtet werden kann und soll. Er muß sich nur vor vordergründigen Gleichsetzungen hüten, die zwar 
möglicherweise politische Vorteile, aber analytisch nur Nachteile einbringen. Bei einer vergleichenden Analyse kommunistischer mit anderen Diktaturen in diesem Jahrhundert erscheinen, nicht erst in der Retrospektive, folgende Aspekte von Bedeutung:

Erstens überstieg die Dauer der Herrschaft der marxistisch-leninistischen Partei, wenn man von Spanien und Portugal absieht, erheblich diejenige anderer Diktaturen. In der Tat haben sie, wie dies Hannah Arendt als typisch für totalitäre Systeme beschrieb, ihre Gründer überlebt und unterschieden sich hierin von faschistischen Bewegungen und vom Nationalsozialismus.

Zweitens unterschied sich der Kommunismus durch die Radikalität seines Bruchs mit der vorausgegangenen »bürgerlichen « Gesellschaft deutlich von anderen diktatorischen Systemen. Alle vorherigen Revolutionen, auch die etwa gleichzeitig mit dem Kommunismus sich etablierenden faschistischen Bewegungen, ließen die alte Eigentums- und Sozialordnung - trotz partieller Veränderungen und korporatistischer Bestrebungen der politischen Führer - weitgehend unangetastet. Insofern waren »Brücken« zur alten Gesellschaft nicht völlig abgebrochen, und es konnte nach dem Ende der Diktatur leichter an deren soziale Muster und Wertorientierung angeknüpft werden - seien es Traditionen der bürgerlichen Gesellschaft, Elemente der ökonomischen oder Verwaltungskultur oder des religiösen Lebens. Der Bruch der kommunistischen Systeme mit diesen Traditionsbeständen war radikaler und dauerhafter als vermutet und stellt eines der wesentlichen Hindernisse für eine schnelle Transition zu Demokratie und Marktwirtschaft in den postkommunistischen Ländern dar (vgl. hierzu Glaeßner 1994).

Im Gefolge der Machtübernahme der kommunistischen Parteien kam es drittens zu einer völligen Umwälzung der Sozialstrukturen. Es wurden nicht nur die politischen und anderen Führungsschichten ausgetauscht, sondern ganze Bevölkerungsgruppen sozial, nicht unbedingt physisch, eliminiert und neue soziale Schichten künstlich geschaffen. Elemente der alten bürgerlichen Gesellschaft blieben allenfalls in Nischen erhalten.

Viertens verhinderte die Zerschlagung der alten sozialen Strukturen und ihre Ersetzung durch eine parteizentralistische Kommando- und nivellierte Sozialstruktur über Jahrzehnte hinweg jede Form einer auch nur rudimentären interessengeleiteten Politik sozialer Gruppierungen und hinterließ nach dem Sturz der kommunistischen Partei ein Vakuum.

Fünftens ist das Paradox zu konstatieren, daß die staatlicher Legitimitätsbeschaffung dienenden sozialstaatlichen Vorstellungen und die Verfestigung egalitärer Ansprüche als wohl einzige Elemente der Ideologie des Marxismus-Leninismus im allgemeinen Wertehaushalt dieser Gesellschaften Wurzeln geschlagen hatten. Angesichts der offenkundigen Ungleichheiten 
in der Sphäre der Macht konnten sie auch als intrasystemares oppositionelles Potential genutzt werden. Nach dem Sturz der kommunistischen Regierungen stellen egalitäre und fürsorgestaatliche Vorstellungen ein erhebliches Resistenzpotential gegenüber einer rein marktwirtschaftlichen und einem allein liberalen Verständnis demokratischer Entwicklung dar.

Und schließlich ist sechstens ein Aspekt des Herrschaftssystems zu erwähnen, der von entscheidender Bedeutung ist: das Fehlen bzw. die Deformation positiven Rechts als normativer Rahmen für das gesellschaftliche Leben während der Diktatur und für die Herausbildung von Werthaltungen, die die Chancen einer Demokratisierung nach der Diktatur wesentlich beeinflussen.

\section{Zwischenresümee - Grenzen der Totalitarismustheorie}

Resümierend läßt sich festhalten, daß die Modellbildungen der Totalitarismustheoretiker zwei Grundpositionen aufweisen: eine eher statische Form, die die Strukturen des Totalitarismus betont, und eine dynamische Sichtweise, die seine Entstehung und Entwicklung in den Mittelpunkt stellt. Eine eindeutige Zuordnung der Autoren zu einer dieser Positionen ist allerdings in der Praxis oft nur schwer möglich. So stützt Franz Neumann seine Aussagen zwar primär auf strukturelle Aspekte (Polizeistaat, Machtkonzentration, monopolistische Staatspartei), ihn interessieren aber vor allem die sozialen Ziele, denen diese Apparaturen ebenso dienen wie der Terror, den sie entfalten. Diese Ziele sind aber nach seiner Auffassung nur zu erkennen, wenn auch das Wirtschaftssystem, die Klassenverhältnisse und die Persönlichkeitsstrukturen in diesen Systemen untersucht werden (Neumann 1977).

Die unterschiedliche Zielorientierung verbietet auch einen einfachen Vergleich von Faschismus und Kommunismus. Statische und dynamische Elemente sind auch bei Sigmund Neumann und Raymond Aron nachweisbar. Selbst im statischen Modell Friedrichs finden sich - zumal durch den Zwang, es an neue Entwicklungen anzupassen - dynamische Elemente, z.B. die These vom Anwachsen des Terrors als konstitutives Kennzeichen totalitärer Herrschaftsausübung.

Die ersten allgemeinen Entwürfe eines Totalitarismusmodells waren dennoch, vergleicht man sie mit den späteren Modifikationen, relativ »eindeutig«. Totalitarismus, bezogen auf »bolschewistische« Regime, war ein allgemeines Modell des Stalinismus. Die Notwendigkeit, dieses Modell zu verändern - das zeigen die Darstellungen von Friedrich und Aron in unterschiedlicher Weise - ergaben sich erst durch den Prozeß der »Entstalinisierung « in der Sowjetunion und den anderen sozialistischen Staaten, der sowohl bestimmte strukturelle Annahmen als auch die Vorstellungen über die 
Richtung dynamischer Prozesse in Frage stellte. Mit den vorgegebenen Antworten des Totalitarismus waren diese Entwicklungen nicht ohne weiteres zu erklären.

Doch wäre es falsch, die Problematik des Totalitarismuskonzepts allein in seiner mangelnden Fähigkeit zu sehen, neue Erscheinungen und Veränderungen zu erklären. Seine entscheidende Problematik liegt in der Identifikation verschiedener idealtypisch gemeinter aber vielfach realtypisch dargestellter Systemtypen (Faschismus, Nationalsozialismus, Kommunismus). Ihre einzige Gemeinsamkeit - und die ist von erheblicher politischer Bedeutung, ist ihre grundsätzlich feindliche Haltung gegenüber der liberalen Demokratie.

Sieht man von der Möglichkeit einer »totalitären Demokratie« ab (Talmon 1961), verbanden sich »identifizierender « Totalitarismusbegriff und »typologische Entgegensetzung « von Totalitarismus, westlicher Demokratie und »Christlichem Abendland «. In der ökonomischen Diskussion fand dies eine besondere Ausprägung in der Dichotomisierung von »totalitärer Planwirtschaft« bzw. »Zentralverwaltungswirtschaft « und freier bzw. sozialer Marktwirtschaft (Kosta 1974; Lane 1976; Pleyer 1969; Thalheim 1965). Zu einem untrennbaren Geflecht verschiedener Argumentationslinien und ebenen verwoben, wurden dabei sowohl die Spezifika der verschiedenen Totalitarismen weitgehend vernachlässigt als auch - und dies ist nicht minder bedeutsam - die besonderen Bedingungen und Ausprägungen innerhalb einzelner Formen des Totalitarismus. Die Vorstellung des totalitären Kommunismus, der in seinem Wesen mit Hilfe eines idealtypischen Modells erfaßt werden sollte, erschwerte nicht nur die Analyse der historischen Entwicklungen und Veränderungen des sowjetischen Systems, sondern eignete sich auch nur mit erheblichen Einschränkungen für die Untersuchung der nach 1945 entstandenen politischen und gesellschaftlichen Systeme in Osteuropa und der DDR, die sich zwar am Vorbild der Sowjetunion orientierten, zugleich aber einer Vielzahl von besonderen Bedingungen unterworfen waren, die von den dargestellten allgemeinen Modellen nicht erfaßt wurden.

Die Möglichkeit einer »Modernisierung « und »Rationalisierung « oder gar eine »Liberalisierung « wurde diesen Systemen im allgemeinen abgesprochen. Nur wenige Totalitarismustheoretiker haben, wie Zbigniew Brzezinski, auf die Möglichkeit hingewiesen, daß sich der Totalitarismus mit spezifisch rationalen Formen politischen und gesellschaftlichen Handelns verbinden könne, ohne daß daraus die Chance einer Demokratisierung erwüchse (vgl. Brzezinski 1962). Durch ihre Fixierung auf Herrschaftsstrukturen und Ideologie hatten die meisten Totalitarismusmodelle ein erkennbares handlungstheoretisches Defizit. Soziales und politisches Handeln unter völlig veränderten Systembedingungen geriet selten in den Blick. 
Szenarien möglicher Krisenentwicklungen waren selten. Die einzige Ausnahme machte $\mathrm{m}$. W. Zbigniew Brzezinski. Er hat in einem Buch, das 1989 unter dem Titel The Grand Failure erschien, die Krise des Kommunismus eindrucksvoll beschrieben und erste Gedanken über mögliche Entwicklungsstränge "postkommunistischer « Systeme angestellt, ohne jedoch ihren kurz bevorstehenden Zusammenbruch zu ahnen - »the book that foretold the collapse of communism in Eastern Europe and Russia «, als das dieses Buch angekündigt wurde, ist es trotz der brillanten Analyse jedoch nicht.

Allerdings hat Brzezinski die Erwartung geäußert, daß das 21. Jahrhundert nicht vom Kommunismus, sondern von der Demokratie geprägt sein werde. $\mathrm{Ob}$ dies als Prognose über den Kollaps des Sowjetimperiums und des von ihm geprägten Systemverbunds anzusehen ist oder eher als eine normative Aussage über einen wünschenswerten zukünftigen Zustand mag dahingestellt bleiben. Brzezinski beantwortete die Frage, was denn aus der von ihm so scharf wie nur von wenigen anderen analysierten Krise des Kommunismus folge, mit zwei grundlegenden, langfristige Alternativen für kommunistische Regime: entweder sie verwandeln sich in eine pluralistische Gesellschaft, oder sie stagnieren innerhalb des gegebenen institutionellen Arrangements und halten ihre Macht durch eine Koalition von Militär und Polizei aufrecht, die anstelle der tradierten Ideologie den Nationalismus als Legitimationsersatz aktiviert. Das erste Szenario hielt Brzezinski für wenig wahrscheinlich. Vieles spreche für einen Übergang vom kommunistischen Totalitarismus zum kommunistischen Autoritarismus und - da dieser sich wesentlich nationalistischer Parolen bediene - zu einem »post-kommunistischen Autoritarismus«. Brzezinski ließ die Frage offen, ob sich die Entwicklung evolutionär oder auf dem Wege eines gewaltsamen Aufbegehrens vollziehen werde.

An vielen Beispielen läßt sich nachweisen, daß oft gerade Totalitarismustheoretiker aus den Grundannahmen ihrer theoretischen Einsichten in das Wesen kommunistischer Systeme geschlossen haben, daß in totalitären Systemen weder eine grundlegende Reform (und hier lag der Dissens zu den verbreiteten Erwartungen der Vertreter des Modernisierungsansatzes) noch ein revolutionärer Umschwung wahrscheinlich seien (Kirkpatrick 1979). Angesichts dieser machtpolitischen Konstellation konnte sich die Kommunismusforschung insgesamt in der Sicherheit wiegen, daß ihr Gegenstand stabil und nur in Grenzen veränderungsfähig sei, weil ein Systembruch nur um den Preis eines Krieges, den vermutlich niemand überleben würde, denkbar erschien. Die in Jahrzehnten entwickelten Sichtweisen, Einschätzungen und Methoden schienen zwar unzulänglich, aber doch brauchbar und hatten von daher Bestand.

Erste Anzeichen eines fundamentalen Wandlungsprozesses konnten noch übersehen oder als Randerscheinung vernachlässigt werden. Aber auch 
nach den radikalen Reformversuchen von Michail Gorbatschow überwog die Einschätzung, daß all dies bestenfalls zu einer lange überfälligen Systemreform führen werde. Völlig außerhalb der Vorstellungskraft aber blieb ein möglicher Kollaps des Kommunismus.

\section{War die DDR totalitär?}

Jedes politische System, ob demokratisch oder autoritär, muß auf die eine oder andere Weise versuchen, die Bindeglieder zwischen politischem System und Gesellschaft institutionell zu verankern und funktionsfähig zu gestalten. Die Inhaber der obersten Macht in autoritären Systemen sind aber bestrebt, diesen Vermittlungsprozeß unter politischer Kontrolle zu halten. Mit Hilfe von »Zwangsorganisationen, die von einer monopolistischen Staatspartei manipuliert werden «, versuchen die modernen Autokratien ihn »auf allen Lebensgebieten, d.h. aber totalitär zu kontrollieren und zu dirigieren « (Fraenkel 1991: 299). Dieser Regelungsanspruch ist zunächst einmal intentional. Auch wenn er als totalitärer Anspruch formuliert wird, konstituiert er noch nicht unbedingt eine totalitäre Wirklichkeit.

Die Entwicklung des politischen Systems der DDR bietet hierfür ein interessantes Exempel. Wesentliches Kennzeichen der politischen Entwicklung in der DDR war der erstaunliche Tatbestand, daß sich die ideologischen Grundlagen des politischen Systems seit dem Ende der 40er Jahre bis zu ihrem Ende im Kern nicht verändert haben. Der Führungsanspruch der SED und die Ideologie des Marxismus-Leninismus sind nie in Frage gestellt worden.

Auf der anderen Seite entfernte sich das politische System schrittweise von der stalinistischen Herrschaft des Terrors, die in der DDR nie so ausgeprägt war wie etwa in der Sowjetunion. Strukturreformen wurden begonnen und wieder verworfen - und am Ende mehr unfreiwillig als bewußt geplant Freiräume für »alternatives « Leben zugelassen. Zugleich aber wurde an den Dogmen des Marxismus-Leninismus festgehalten und allen Vorstellungen von einer Pluralisierung und teilweisen demokratischen Öffnung des Systems eine klare Absage erteilt. Es blieb der totalitäre Anspruch der Parteiherrschaft. Es blieb bei der Ablehnung der liberalen Demokratie und rechtsstaatlicher Normen und Verfahren.

Die Etablierung des Sozialismus in der Sowjetischen Besatzungszone und den mittel-, ost- und südosteuropäischen Ländern nach 1945 war, von Jugoslawien abgesehen, nicht das Ergebnis einer innergesellschaftlichen Transformation kapitalistischer Produktionsverhältnisse, sondern Fernwirkung der in Jalta beschlossenen Aufteilung Europas in Einflußzonen der Großmächte, zu denen in der Folge des II. Weltkrieges auch die Sowjetunion gestoßen war. Der Sozialismus, der im objektivistisch-deterministischen 
Geschichtsverständnis des Marxismus-Leninismus notwendiges Ergebnis eines historischen Prozesses, nämlich die positive Aufhebung des Kapitalismus und kurze Übergangsphase zum Kommunismus sein sollte, etablierte sich nach 1945 als auf Dauer angelegtes Herrschaftssystem und Antipode zum fortbestehenden Kapitalismus, der anders als erwartet, keine Neigung zeigte unterzugehen. Die Stalinsche Reduktion der Wirklichkeit auf die weltweite Auseinandersetzung zwischen Sozialismus und Imperialismus konnte »Dritte Wege« weder denken noch zulassen. Drei Aspekte prägten diesen Sozialismus sowjetischen Typs, der mit dem demokratischen Sozialismus westeuropäischer Prägung nur noch den Namen gemeinsam hatte:

Erstens: In den industriell wenig oder nicht entwickelten Ländern (Rußland/Sowjetunion, China, Korea, Jugoslawien, Albanien, Rumänien, Bulgarien, später in Kuba und Vietnam) etablierte sich dieser Sozialismus als Modernisierungssystem. Er setzte sich das Ziel, die Industrialisierung eines zurückgebliebenen Landes durch eine gewaltige, von der kommunistischen Partei initiierte und gelenkte Anstrengung in kurzer Zeit nachzuholen. Dies forderte ungeheure menschliche und soziale Opfer. Die kommunistische Partei konnte dabei kaum auf die Unterstützung einer Bevölkerung rechnen, die durch diesen Prozeß sozial und kulturell entwurzelt wurde.

Zweitens: In den Ländern Mittel- und Osteuropas (mit der Ausnahme Jugoslawiens) präsentierte sich der Sozialismus als Okkupationsregime. Das ökonomische und politische System der Sowjetunion wurde - mit geringfügigen Abwandlungen - sowohl auf »moderne « Länder wie die DDR oder die Tschechoslowakei als auch auf noch nicht voll entwickelte Gesellschaften wie Bulgarien oder Rumänien übertragen, ohne die völlig verschiedenen sozial-ökonomischen, kulturellen und historischen Voraussetzungen in Rechnung zu stellen. Auf der Ebene zwischenstaatlicher Beziehungen wurde das Verhältnis der Vorkriegs-KPs zur KPdSU und zur III. Internationale reproduziert. Die Sowjetunion war die politische, ökonomische, militärische und ideologische Führungsmacht.

Drittens: $\mathrm{Ob}$ es sich um industriell entwickelte oder zurückgebliebene Länder handelte, die marxistisch-leninistische Transformationsvorstellung bot für die unterschiedlichen Gesellschaften nur eine Strategie an: die bürokratisch-zentralistische Regulierung aller politischen, wirtschaftlichen und sozialen Beziehungen durch die marxistisch-leninistische Partei, die sich selbst als Avantgarde begriff.

Vor diesem Hintergrund stellt sich die Frage nach der historischen Legitimation der Systeme des »realen Sozialismus «. Sie waren entstanden und übten ihre Herrschaft aus, weil sie ein politisches Ziel zu verwirklichen trachteten. Insoweit waren sie Zielkulturen (»goal cultures«). Es ging ihnen um die Errichtung einer kommunistischen Gesellschaft, in der die Herr- 
schaft von Menschen über Menschen abgeschafft und eine neue politische und gesellschaftliche Kultur verwirklicht werde. Die kommunistischen Parteien repräsentierten Bewegungen, welche die politische und gesellschaftliche Kultur transformieren wollten (»culture transforming movements«).

In ihrer eigenen Vorstellung war Politik ein Prozeß, in dem die Ziele und der Wille der herrschenden Klasse, des Proletariats, auf die gesamte Gesellschaft übertragen werden sollte. Politische Prinzipien und Entscheidungen wurden nicht als das Ergebnis eines konfliktreichen Austrags und Ausgleichs unterschiedlicher Interessen, sondern als Verwirklichung der historischen Mission der Arbeiterklasse verstanden. Diese monistische Vorstellung von Politik beruhte auf der ideologisch präformierten Prämisse, daß der Marxismus-Leninismus mit seiner exklusiven Einsicht in die Entwicklungsgesetze der menschlichen Gesellschaft in der Lage sei, die objektiven Gesetzmäßigkeiten der Geschichte zu erkennen und daraus die »richtigen« Schlußfolgerungen zu ziehen.

Die Verwirklichung des gesellschaftspolitischen Ziels, unter der Führung der Arbeiterklasse und ihrer marxistisch-leninistischen Partei den Sozialismus und schließlich die kommunistische Gesellschaft zu verwirklichen, bedurfte nach Auffassung der SED einer starken, einheitlichen Führung und einer nach einheitlichen Prinzipien gestalteten Politik. Dieser allumfassende Führungsanspruch der Partei fand seinen Niederschlag in einer hyperzentralisierten Struktur des politischen Systems. Politik, Wirtschaft und Verwaltung waren ebenso wie alle anderen gesellschaftlichen Vollzüge einem einheitsstiftenden Prinzip unterworfen - dem »demokratischen Zentralismus«. Mit seiner Hilfe setzten die Parteiführungen ihren Willen innerhalb der kommunistischen Parteien selbst und gegenüber der Gesamtgesellschaft durch.

Der demokratische Zentralismus, im zaristischen Rußland von den in der Illegalität operierenden Bolschewiki entwickelt und von Lenin in seiner Schrift Was tun? aus dem Jahre 1902 theoretisch begründet, war in der Zwischenkriegszeit zum herrschenden Organisationsprinzip der Parteien der Kommunistischen Internationale geworden und wurde nach dem II. Weltkrieg in den sozialistischen Ländern von einem innerparteilichen zu einem Strukturprinzip der gesamten Gesellschaft erhoben. Der demokratische Zentralismus schuf eine dichotomische Struktur der Gesellschaft. Die Partei stand als führende Kraft den Bürgern gegenüber, von denen erwartet wurde, die von der Partei formulierten Ziele zu ihren eigenen zu machen. Er stellte zudem ein hierarchisches Verhältnis zwischen der Partei (bzw. dem Parteiapparat) und den übrigen »gesellschaftlichen Organisationen « her. Verbände und Vereinigungen, die das Alltagsleben der Menschen mitgestalteten, waren keine eigenständigen Organisationen, die wie in einer pluralistischen Gesellschaft dem Willen ihrer Mitglieder Gehör verschaffen 
und ihre Interessen gegenüber der Politik vertreten sollten, sondern hatten als »Transmissionsriemen « den Willen der Partei in allen Bereiche der Gesellschaft umzusetzen - sei es in der Arbeitswelt, im Bildungswesen, der Wissenschaft oder Kultur.

Ein ebenso instrumentelles Verständnis hatte die SED auch von den Aufgaben und Funktionen des Staates. Er sollte nicht mehr, aber auch nicht weniger sein, als das »Hauptinstrument « der Arbeiterklasse und ihrer marxistisch-leninistischen Partei, mit dessen Hilfe sie ihre politischen und gesellschaftlichen Ziele zu verwirklichen gedachte. Politik war im Verständnis des SED Staatspolitik. Das war nicht selbstverständlich angesichts einer Theorie, die sich auf Marx und Engels berief, deren Vision es gewesen ist, den Staat als Unterdrückungsinstrument abzuschaffen, nicht aber einer neuen, mit allen Machtmitteln ausgestatteten und demokratisch nicht legitimierten und kontrollierten Führung zu überlassen. Der MarxismusLeninismus beschrieb diesen neuen Typus von Staat als »Diktatur des Proletariats « - Vorbild war die Sowjetunion.

Im politischen Verständnis der SED sollte der Staat die Gesellschaft organisatorisch, die Partei sie politisch-ideologisch zusammenhalten. Der Staat mußte parteilicher Staat sein und sich in allen seinen Handlungen vom Willen und den Absichten der Partei leiten lassen. Dies bedeutete, daß die SED durch organisatorische Vorkehrungen dafür zu sorgen hatte, daß staatliche Institutionen kein »Eigenleben « entwickeln konnten. Das Prinzip des demokratischen Zentralismus und die Prinzipien der Kaderpolitik garantierten, daß die »staatlichen Organe « vollständig von der SED abhängig waren und die Weisungen der Partei ausführten.

Zwar gab es auch im politischen System der DDR eine funktionale Aufgabenverteilung zwischen Partei, Exekutive, Legislative und Judikative, zwischen staatlichen Institutionen und "gesellschaftlichen Organisationen « sowie hierarchisch gestaffelte Kompetenzzuweisungen an die regionalen Untergliederungen (Bezirke, Kreise, Städte und Gemeinden). Allerdings wurde die Prärogative der Partei und ihr prinzipielles Recht, jederzeit in staatliche Angelegenheiten und die Belange der Gesellschaft einzugreifen, niemals ernsthaft in Frage gestellt. Das Organisationsgefüge des »realen Sozialismus «, das alle gesellschaftlichen Bereiche umspannte, war Ausdruck einer mechanistischen Konzeption von Politik. Trotz aller Versuche, sich am Muster moderner Organisationsvorstellungen zu orientieren, blieb die marxistisch-leninistische Organisationslehre stets ihrer Herkunft aus geheimbündlerischen Vereinigungen verhaftet und hatte Mühe, sich nach rationalen Kriterien zu organisieren (Ludz 1970: 25ff).

Durch die Vereinigung von staatlichem Eigentum an den Produktionsmitteln und staatlicher Bürokratie entstand eine Herrschaftsstruktur, die grundsätzlich keine konkurrierenden Ziele kannte (Hegedüs 1981: 77). Jahrzehn- 
telang wurde der extreme Zentralismus und der Mangel an Demokratie damit gerechtfertigt, daß nur so eine ökonomisch leistungsfähige und sozial gerechte sozialistische Gesellschaft aufgebaut werden könne. In den 70er und 80er Jahren wurde offenkundig, daß die realsozialistischen Systeme immer weniger in der Lage waren, diese Ziele zu verwirklichen und die notwendigen Modernisierungsprozesse voranzutreiben.

Zwischen dem umfassenden Führungsanspruch der Partei auf der einen und den Anforderungen einer komplexen industriell entwickelten Gesellschaft auf der anderen Seite, die ohne eine strukturelle Differenzierung, die Berücksichtigung von Rationalitäts- und Effektivitätskriterien in der zentral gelenkten und geplanten Wirtschaft und ohne eine minimale Beteiligung der Bürger an den gesellschaftlichen Prozessen nicht auskommen konnte, gab es eine unüberwindbare Kluft. Der Kern und die eigentliche Ursache aller politischen und sozialen Krisen in den Ländern Osteuropas und der DDR war die Tatsache, daß die Sowjetunion auf Grund der nach 1945 entstandenen militärischen und geopolitischen Konstellation die Chance erhielt, ihren neuen Satelliten (oder Verbündeten) ein politisches, soziales und wirtschaftliches System überzustülpen, das für ganz andere sozialökonomische Ziele (die der Modernisierung und Industrialisierung eines zurückgebliebenen Landes) und eine ganz andere politische Kultur gedacht war. Die industriell entwickelten Staaten, insbesondere die DDR und die Tschechoslowakei standen also von Beginn vor dem Problem, daß ihnen politische und ökonomische Strukturen oktroyiert wurden, die für ein Land konzipiert waren, in dem es galt, die Industrialisierung unter »sozialistischen« Vorzeichen nachzuholen. Es war das Modell eines Modernisierungssystems mit totalitärem Anspruch, das zum Vorbild der sozialistischen Umgestaltung in der SBZ/DDR wurde. Für ein industriell entwickeltes, wenngleich durch den Krieg zerstörtes Land, bedeutete dies einen historischen Rückschritt mit weitreichenden politischen, wirtschaftlichen und sozialen Folgen.

Die Frage, ob die DDR »totalitär « war, läßt sich nicht isoliert beantworten. Als Teil eines Systemsverbunds, dessen Grundstrukturen durch die Sowjetunion in der Zeit des Stalinismus oktroyiert worden waren und die im Verlauf der Jahrzehnte keine grundlegende Reform erfahren haben - alle diesbezüglichen Ansätze, vor allem in den frühen 60er Jahren, wurden nach kurzer Experimentierzeit aus Furcht vor der Gefährdung der »führenden Rolle der Partei« zurückgenommen oder verwässert -, war sie eine Herrschaftsordnung, in der eine Partei, mit dem Verweis auf ihre historische Mission und die objektive Erkenntnis der »historischen Gesetzmäßigkeiten« den Anspruch erhob, alle Bereiche der Gesellschaft nach ihrem Willen zu gestalten. Insofern, aber auch nur insofern, war die DDR totalitär.

Ein »intentionaler Totalitarismus « ist aber keineswegs mit einem praktisch 
realisierten Totalitarismus gleichzusetzen. Allenfalls in den Anfangsjahren der SBZ/DDR waren die Strukturelemente ausgeprägt und die politische Führung willens und fähig, ihren Anspruch mit allen Machtmitteln, d.h. auch mit Terror durchzusetzen. Mit der »Veralltäglichung « der Revolution, der Verfestigung der neuen politischen, wirtschaftlichen und sozialen Strukturen und mit der Dauer der Herrschaft der marxistisch-leninistischen Partei schliffen sich die totalitären Elemente der Herrschaftsordnung ab obwohl sie bis zum Ende des Systems, wie die vielfältigen, 1989 nicht realisierten »Notstandspläne « zeigen, als Drohkulisse für politische und gesellschaftliche Krisenzeiten erhalten blieben.

\section{Literatur}

Arendt, Hannah (1986): Elemente und Ursprünge totaler Herrschaft, München: Piper.

Aron, Raymond (1970): Demokratie und Totalitarismus, Hamburg: Christian Wegner Verlag.

Bracher, Karl Dietrich (1985): Zeit der Ideologien. Eine Geschichte politischen Denkens im 20. Jahrhundert, München: dtv.

Brzezinski, Zbigniew (1962): Der Sowjetblock. Einheit und Konflikt, Köln/Berlin: Kiepenheuer \& Witsch.

- (1989): The Grand Failure. The Birth and Death of Communism in the Twentieth Century, New York: Collier Books (2. Aufl. 1990)

Fraenkel, Ernst (1991): Deutschland und die westlichen Demokratien, Frankfurt a. M.: Suhrkamp.

Friedrich, Carl Joachim unter Mitarbeit von Zbigniew Brzezinski (1957): Totalitäre Diktatur, Stuttgart: Kohlhammer.

Friedrich, Carl Joachim (1970): Politik als Prozeß der Gemeinschaftsbildung. Eine empirische Theorie, Köln/Opladen: Westdeutscher Verlag (engl.: Man and His Government. An Empirical Theory of Politics, New York: McGraw-Hill 1963).

Furet, Francois (1995): Das Ende der Illusion. Der Kommunismus im 20. Jahrhundert, München: Piper.

Glaeßner, Gert-Joachim (1982): Sozialistische Systeme. Einführung in die Kommunismus- und DDR-Forschung, Opladen: Westdeutscher Verlag.

- (1994): Demokratie nach dem Ende des Kommunismus. Regimewechsel, Transition und Demokratisierung im Postkommunismus, Opladen: Westdeutscher Verlag.

- (1995): Kommunismus - Totalitarismus - Demokratie. Studien zu einer säkularen Auseinandersetzung, Frankfurt a.M.: Peter Lang.

Hassner, Pierre (1985): Communist Totalitarianism: The Transatlantic Vagaries of a Concept

Hegedüs, András (1981): Sozialismus und Bürokratie, Reinbek: Rowohlt.

Hildebrand, Klaus (1968): Stufen der Totalitarismusforschung, in: Politische Vierteljahresschrift, 9. Jg./1968, Nr. 3, S. 397-422.

Hobsbawm, Eric (1994): Age of Extremes. The Short Twentieth Century 1914-1991. London: Michael Joseph.

Huntington, Samuel P. (1991): The Third Wave: Democratization in the Late Twentieth Century. Norman: University of Oklahoma Press.

Kirkpatrick, Jeane (1979): Dictators and Double Standards, in: Commentary, Vol. 68 (November 1979), S. 34-45.

Kosta, Ji• i (1974): Sozialistische Planwirtschaft. Theorie und Praxis, Opladen: Westdeutscher Verlag.

Lane, David (1976): The Socialist Industrial State. Towards a Political Sociology of State Socialism, London: Allen \& Unwin.

Linz, Juan J. (1975): Totalitarian and Authoritarian Regimes, in: Greenstein, Fred I./Nelson W. Polsby (Eds.): Handbook of Political Science, Bd. 3: Macropolitical Theory, Reading, 
Mass.: Addison-Wesley, S. 175-411.

Löwenthal, Richard (1964): World Communism: The Desintegration of a Secular Faith, New York/Oxford: Oxford University Press.

Ludz, Peter Christian (1970): Parteielite im Wandel. Funktionsaufbau, Sozialstruktur und Ideologie der SED-Führung. Eine empirisch-systematische Untersuchung, 3. Aufl., Köln/Opladen: Westdeutscher Verlag.

Neumann, Franz Leopold (1977): Behemoth. Struktur und Praxis des Nationalsozialismus, Frankfurt a. M.: EVA.

Neumann, Sigmund (1965): Permanent Revolution. Totalitarianism in the Age of International Civil War, London: Pall Mall Press.

Pleyer, Klemens/Joachim Lieser (1969): Zentralplanung und Recht, Stuttgart: Fischer.

Shapiro, Leonard (1969): The Concept of Totalitarianism, in: Survey. A Journal of Soviet and East European Studies, No. 73 (Autumn 1969), S. 93-115.

Talmon, Jacob Leib (1961): Die Ursprünge der totalitären Demokratie, Köln/Opladen: Westdeutscher Verlag.

Thalheim, Carl C. (1965): Beiträge zur Wirtschaftspolitik und Wirtschaftsordnung, Berlin: Duncker \& Humblodt. 